\title{
Effective Evaluation of the Noise Factor of Microchannel Plate
}

\author{
Honggang Wang, ${ }^{1,2}$ Yujie Du, ${ }^{2}$ Yu Feng, ${ }^{1}$ Yang $\mathrm{Lv},^{2}$ Xiaoming Hu, ${ }^{2}$ and Yunsheng Qian ${ }^{2}$ \\ ${ }^{1}$ School of Information and Electrical Engineering, Ludong University, Yantai 264025, China \\ ${ }^{2}$ School of Electronic and Optical Engineering, Nanjing University of Science and Technology, Nanjing 210094, China
}

Correspondence should be addressed to Honggang Wang; whgwwl@163.com

Received 13 June 2015; Accepted 13 August 2015

Academic Editor: Jung Y. Huang

Copyright (C) 2015 Honggang Wang et al. This is an open access article distributed under the Creative Commons Attribution License, which permits unrestricted use, distribution, and reproduction in any medium, provided the original work is properly cited.

\begin{abstract}
To improve the noise performance of microchannel plate (MCP), we have presented a method using the sine random signals with Poisson distribution as the noise-excitation for electron source. By using this method, the effective evaluation of noise characteristics of MCP has been implemented through measuring and analyzing its noise factor. The results have demonstrated that the noise factor of filmed MCP is lower than 1.8. Additionally, as the open area ratio and the input electron energy are $72 \%$ and $400 \mathrm{eV}$, respectively, the noise characteristics of unfilmed MCP are improved evidently. Moreover, larger open area ratio, higher input electron energy, and higher voltage across the MCP all can reduce effectively the noise factor within a certain range. Meanwhile, the ion barrier film extends the life of image tube but at the cost of an increased noise factor. Therefore, it is necessary that a compromise between the optimum thickness of ion barrier film, open area ratio, input electron energy, and voltage across the MCP must be reached.
\end{abstract}

\section{Introduction}

Modern photoelectric imaging intensifiers for various applications often employ a microchannel plate (MCP) to generate electronic gain by secondary electron multiplication [1]. It is interesting to investigate the noise performance of the MCP because our image intensifiers are affected by poor signalto-noise ratio (SNR). By researching the noise characteristics of the critical component of the system, that is, MCP, it is possible to optimize the image intensifier design to the lowest noise levels possible. Since the MCP is used in imaging applications as an amplifier, the concept of a noise factor $\left(N_{F}\right)$ is appropriate [2].

For the last decades, $N_{F}$ has been generally used as a criterion of evaluating the MCP noise characteristics, and further a substantial improvement is reduced $N_{F}$ [3-7]. Therefore, it is very important to effectively evaluate $N_{F}$ of MCP in image intensifiers. Currently, the noise characteristics of entire image intensifier, the effect of parameters of $\mathrm{MCP}$ on image intensifier performance, and the fabrication of MCP have mainly been concerned, whereas little attention has been paid to the noise characteristics of MCP itself [8-11]. In addition, the disadvantage of results presented in [7] is that the values of input SNR and output SNR are obtained by constant filament current, which are not consistent with the condition of MCP operating in image intensifier. Moreover, according to the requirements of $N_{F}$ measurement, the bottleneck is the significant difference between very low input current density $\left(10^{-11}-10^{-10} \mathrm{~A} / \mathrm{cm}^{2}\right)$ and high SNR, which leads to the fact that the input current noise is overwhelmed by the ambient interference noise, and then the measurement becomes difficult. To address this problem, we present a method using the sine random signals with Poisson distribution as the noise-excitation for electron source, through which the varying filament current satisfying the measurement requirements is generated. Accordingly, the results agree with the practical condition of MCP; in other words, the objective evaluation of the noise characteristics of MCP can be achieved by determining $N_{F}$, which has been experimentally verified.

\section{Evaluation Method}

2.1. Noise Factor. From a practical standpoint, since the number of electrons injected into MCP is random, the noise caused by the fluctuation in these electrons is the input noise. Accordingly, the signal-to-noise ratio at the input end $\left(\mathrm{SNR}_{\mathrm{in}}\right)$ of $\mathrm{MCP}$ is defined as the ratio of the input 
average signal to the root-mean-square (RMS) deviation from its mean value. Besides, the signal-to-noise ratio at the output end $\left(\mathrm{SNR}_{\text {out }}\right)$ is formed by the imperfection of MCP itself and the statistical nature of the gain process in $\mathrm{MCP}$. Correspondingly, $\mathrm{SNR}_{\text {out }}$ is expressed as the ratio of the output average signal to the root-mean-square (RMS) deviation from its mean value. It is worth noting that the noise characteristic of MCP is affected not only by that of MCP itself but also by $\mathrm{SNR}_{\mathrm{in}}$, although this characteristic can be appraised through $\mathrm{SNR}_{\text {out }}$. Hence, $N_{F}$ of MCP is defined as [2]

$$
N_{F}=\frac{\mathrm{SNR}_{\text {in }}}{\mathrm{SNR}_{\text {out }}} .
$$

From formula (1), it can be seen that the deterioration of SNR caused by MCP itself can be represented with $N_{F}$ due to eliminating the impact of $\mathrm{SNR}_{\mathrm{in}}$, and thus the noise characteristic of MCP is analyzed objectively. Concretely, smaller $N_{F}$ stands for better performance of noise suppression.

\subsection{Implementation of the Electron Beam Incorporating Noise-} Excitation. The electron source plays an important role in the measuring system, and it generates electron beam to be accelerated by an electric field to the MCP. In fact, the electrons are given off from a photocathode at random times, and their distribution for number of electrons generated per unit time interval is governed by Poisson distribution. To be more consistent with the actual work conditions of image intensifier, we use a group of sinusoidal signals with Poisson distribution as the noise-excitation, thereby generating the electron beam incorporating noise-excitation required by $N_{F}$ measurement. In the following, the generation of these signals is described.

To begin with, a random vector with Poisson distribution is generated, and it takes the form of

$$
X_{0}=\left[x_{0}, x_{1}, \ldots, x_{N-1}\right]^{T},
$$

where, here, $T$ stands for the transpose operation. Second, to meet the requirements of harmonic coefficient, a new even symmetry vector must be constructed on the basis of $X_{0}$, and it is given by

$$
X=\left[x_{N-1}, x_{N-2}, \ldots, x_{0}, x_{0}, x_{1}, \ldots, x_{N-1}\right]^{T} .
$$

Furthermore, the elements of $X$ are expressed as

$$
\begin{aligned}
& x(n), \\
& (n=-(N-0.5),-(N-1-0.5), \ldots,-0.5,0.5, \ldots,(N-0.5)),
\end{aligned}
$$

where, here, $n$ represents the time index in time domain. And then, the sampling interval in frequency domain is set as $\Delta \omega=2 \pi / 2 N$, for obtaining the coefficients and frequencies of harmonic. Correspondingly, the coefficient of $k$ th harmonic is given by

$$
F(k)=\sum_{n=-(N-0.5)}^{N-0.5} x(n) e^{-j \cdot \Delta \omega \cdot k \cdot n},
$$

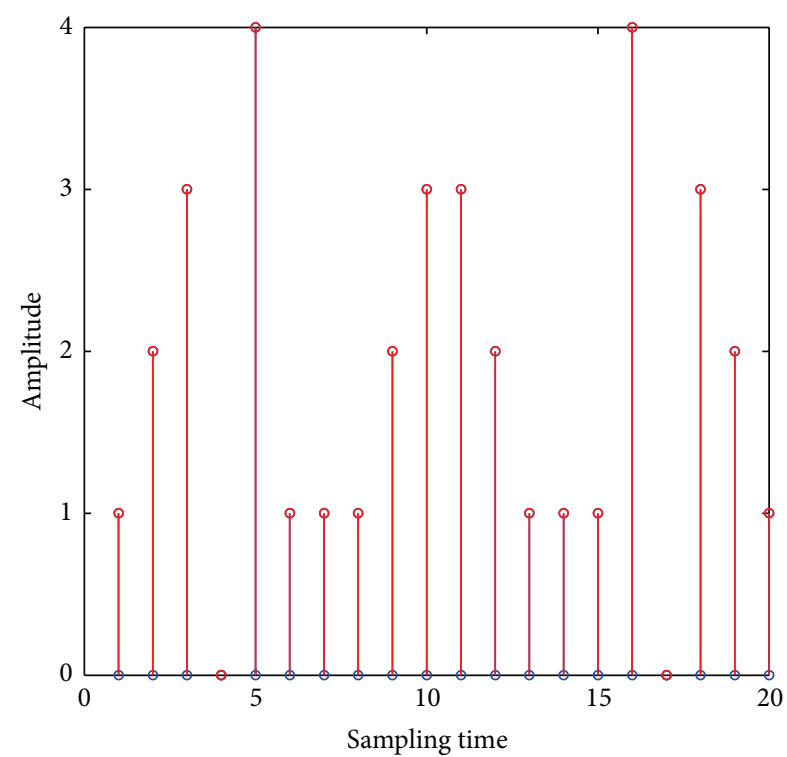

Figure 1: The sine random signals with Poisson distribution.

where $k$ denotes the normalized frequency index. From formula (5), we can obtain

$$
\begin{aligned}
F(k) & =\sum_{n=0.5}^{N-0.5} x(-n) e^{j \cdot \Delta \omega \cdot k \cdot n}+\sum_{n=0.5}^{N-0.5} x(n) e^{-j \cdot \Delta \omega \cdot k \cdot n} \\
& =2 \sum_{n=0.5}^{N-0.5} x(n) \cos (\Delta \omega \cdot n \cdot k) .
\end{aligned}
$$

Finally, the sinusoidal signals obeying Poisson distribution as the noise-excitation can be obtained through the expression of

$$
f(n)=\frac{1}{N} \sum_{n=0.5}^{N-0.5} F(k) \cos (\Delta \omega \cdot n \cdot k) .
$$

Subsequently, the sine random signals with Poisson distribution are shown in Figure 1.

According to formula (7), the principle of electron source system which generates the input electron beam incorporating noise-excitation is shown in Figure 2. This system consists of a microprocessor unit, a drive power supply, an electron gun, and an electrooptical system. With the appropriate algorithm, the microprocessor accepts the instructions sent by computer control software and then generates the electron beam satisfying the measurement requirements.

\section{Experimental Results}

3.1. Measuring Setup. A schematic diagram of $N_{F}$ measuring system of MCP is shown in Figure 3. The workflow of this system is as follows. First, according to the instruction sent by the industrial processing (IP) computer, an actuator can control the mechanical pump and the molecular pump to vacuumize the chamber. Second, the high voltage power supply provides the appropriate voltages for the electron gun, 


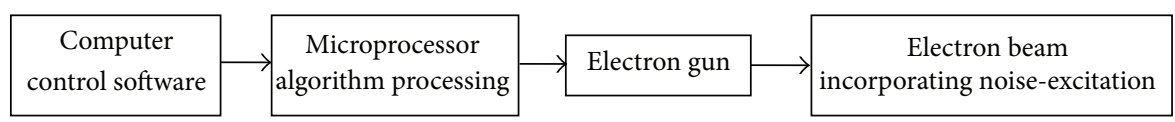

FIGURE 2: The principle diagram of electron source incorporating noise-excitation.

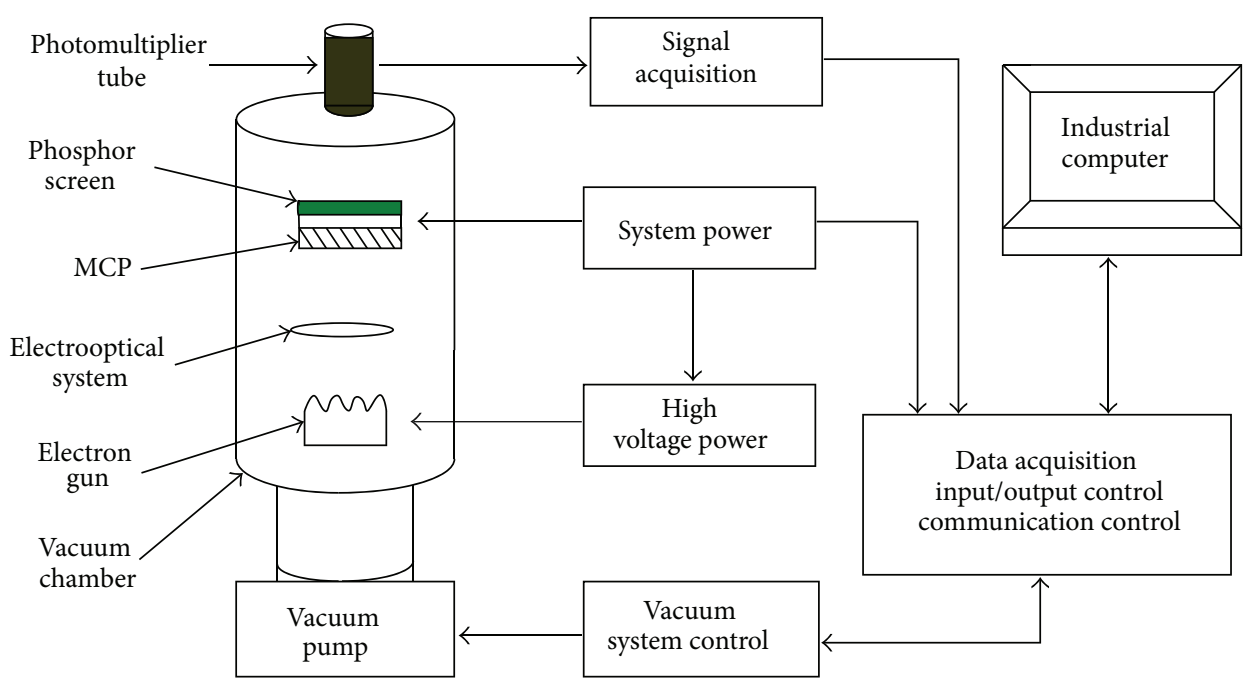

FIGURE 3: Schematic diagram of the noise factor measurement system of MCP.

the electron-optical system, and the MCP with fluorescent screen. Correspondingly, the electron beam incorporating noise-excitation will be injected into the input end of MCP and then be multiplied to bombard the screen. Finally, using a low dark current photomultiplier and the signal acquisition processing units, the output signal is detected and then processed. Furthermore, with the help of the test software, $N_{F}$ of MCP can be calculated.

3.2. Measuring Conditions. To scientifically evaluate the noise characteristics of MCP, it is necessary to determine the appropriate measuring conditions. These conditions mainly include the current density of input electron beam, the diameter of electron beam, the noise band width, and vacuum degree. In terms of the work conditions of MCP and measuring system, the values of these conditions are given as follows:

(1) Current density of input electron beam: $1 \times 10^{-11}-1 \times$ $10^{-10} \mathrm{~A} / \mathrm{cm}^{2}$; filament current: $2100-2300 \mathrm{~mA}$.

(2) Diameter of electron beam: $16 \mathrm{~mm}$.

(3) Noise band width: $0-10 \mathrm{~Hz}$.

(4) Vacuum degree: superior to $5 \times 10^{-4} \mathrm{~Pa}$.

3.3. Results and Discussion. In accordance with the above measuring conditions, we have determined the value of $\mathrm{SNR}_{\text {in }}$ as 48.54 and measured $N_{F}$ of various MCP, including BB (employed in generation super II, channel diameter of $6 \mu \mathrm{m}), \mathrm{BF}$ (employed in generation III, channel diameter of
$6 \mu \mathrm{m}$ ), and BC (employed in generation III, channel diameter of $5 \mu \mathrm{m})$. The measurement results are as follows.

3.3.1. Noise Factor of Unfilmed MCP and Filmed MCP. By using the identical measuring conditions, $N_{F}$ of unfilmed MCP and MCP coated at the input surface with ion barrier film (IBF, $\mathrm{Al}_{2} \mathrm{O}_{3}$ ) have been obtained and shown in Table 1 .

As illustrated in Table $1, N_{F}$ of MCP coated with IBF is higher than that of unfilmed MCP. Apparently, this is directly related to the scattering and resisting effects of IBF on the photoelectrons emitted from the photocathode. An intrinsic feature of an operating MCP is the desorption and ionization of residual gases in the channels of the plate. The internal electric field of the MCP will accelerate these positive ions toward the input plane of the MCP. For the thirdgeneration image tubes, if these ions are allowed to escape from the MCP and interact with the GaAs photocathode, they can cause irreversible damage to the cesium and oxygen (Cs-O) activation layer causing rapid decay in the cathode photoresponse. Furthermore, the decay in photoresponse has manifested itself as a reduction in $\mathrm{SNR}_{\text {out }}$ [12]. To circumvent this problem, it has been standard practice in the industry to employ the MCP that is covered with a thin $\mathrm{Al}_{2} \mathrm{O}_{3}$ or $\mathrm{SiO}_{2}$ film to prevent ion feedback from the MCP to the photocathode. The thickness of IBF is determined by the available cathode-to-MCP bias and the penetration voltage of the film. For maximum transmission of electrons through the IBF, it is necessary to apply a higher potential difference between photocathode and MCP than would otherwise be required if the MCP were unfilmed. While the application of an IBF to the MCP greatly improves the mean time to 
TABLE 1: Noise factor of unfilmed and filmed MCP.

\begin{tabular}{lccccc}
\hline Samples & SNR $_{\text {in }}$ & SNR $_{\text {out }}$ (unfilmed) & SNR $_{\text {out }}$ (filmed) & $N_{F}$ (unfilmed) & $N_{F}$ (filmed) \\
\hline BF0120-026-155 & 48.54 & 33.32 & 29.67 & 1.457 & 1.441 \\
BF0120-026-157 & 48.54 & 33.68 & 31.54 & 1.4836 \\
BF0120-026-166 & 48.54 & 32.71 & 30.84 & 1.468 & 1.539 \\
BF0120-026-170 & 48.54 & 33.07 & 29.35 & 1.574 \\
BF0120-026-172 & 48.54 & 31.34 & 27.24 & 1.654 \\
\hline
\end{tabular}

TABLE 2: Noise factor of MCP with different open area ratios.

\begin{tabular}{lccc}
\hline Samples & SNR $_{\text {in }}$ & SNR $_{\text {out }}$ & $N_{F}$ \\
\hline BB4031-109-420 (64\%) & 48.54 & 34.42 & 1.410 \\
BB4061-281-445 (72\%) & 48.54 & 38.78 & 1.252 \\
BC2032-006-111 (64\%) & 48.54 & 37.56 & 1.292 \\
BC2032-007-162 (64\%) & 48.54 & 38.69 & 1.255 \\
BC4060-003-123Y (72\%) & 48.54 & 42.07 & 1.154 \\
BC4060-003-150Y (72\%) & 48.54 & 41.83 & 1.160 \\
\hline
\end{tabular}

failure of the image tube, it unfortunately causes significant degradation in $\mathrm{SNR}_{\text {out }}$ of the MCP and thus leads to increased $N_{F}$.

3.3.2. Noise Factor of MCP with Different Open Area Ratios. To determine the effect of the open area ratio on noise factor, $N_{F}$ of MCP with an open area ratio of $64 \%$ and with an open area ratio of $72 \%$ are measured, respectively. The measurement results are shown in Table 2.

As shown in Table 2, it is obvious that whether BB or BC, $N_{F}$ of MCP with an open area ratio of $72 \%$ is superior to that of MCP with an open area ratio of $64 \%$. In reality, the MCP consisting of a parallel array of glass channels has an open area ratio of less than $100 \%$; thus electrons are lost or scattered when they strike the interchannel boundaries. The scattered electrons will cause signal generated noise and a reduction in $\mathrm{SNR}_{\text {out }}$, that is, an increase in $N_{F}$. Additionally, the theoretical decrease in $N_{F}$ with increasing open area ratio has been given in the literature [13]. Hence, the input open area ratio becomes an important feature of MCP for use in image intensifier tubes. Fortunately, funneling of the input surface of MCP increases the input electron collection efficiency through increasing open area ratio and thus reduces $N_{F}$ of MCP.

3.3.3. Noise Factor of MCP at Different Input Electron Energies. The second electron yield of MCP is another important characteristic which governs $N_{F}$ of MCP. When a primary event impacts the channel input side, secondary electrons may be generated. The number of secondary electrons generated is determined primarily by the energy and angle of incidence of the primary event. Therefore, for a given unfilmed MCP, to further discuss the effect of input electron energy on noise factor, we have measured its $N_{F}$ in the range of $100-700 \mathrm{eV}$. The results are given in Table 3; additionally, Figures 4 and 5 show the effect of input electron energy on $N_{F}$ and the rate of increase in signal $(S)$, noise $(N)$, and $\mathrm{SNR}_{\text {out }}$, respectively.

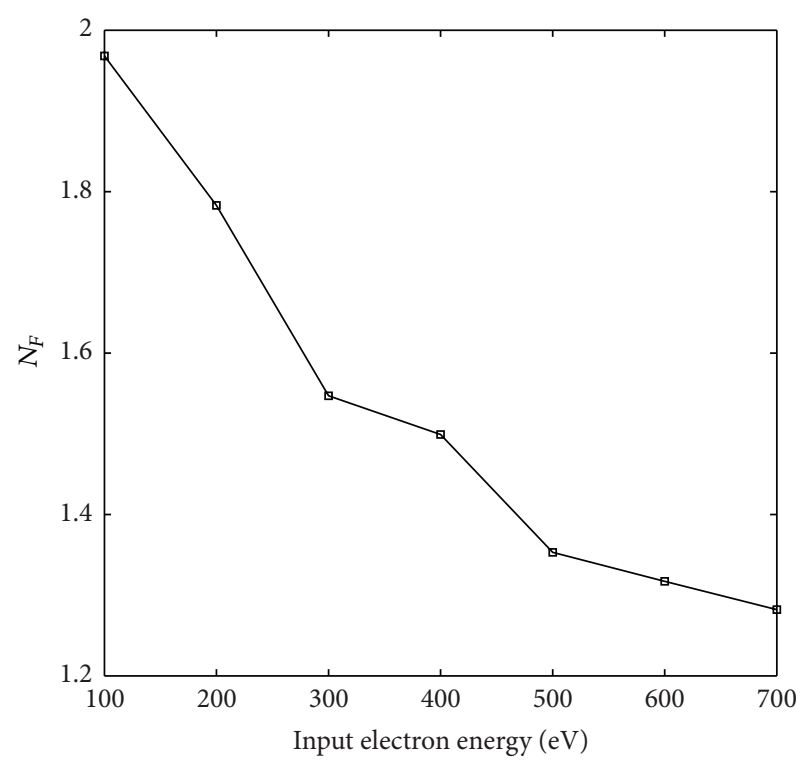

Figure 4: $N_{F}$ of MCP at different input electron energies.

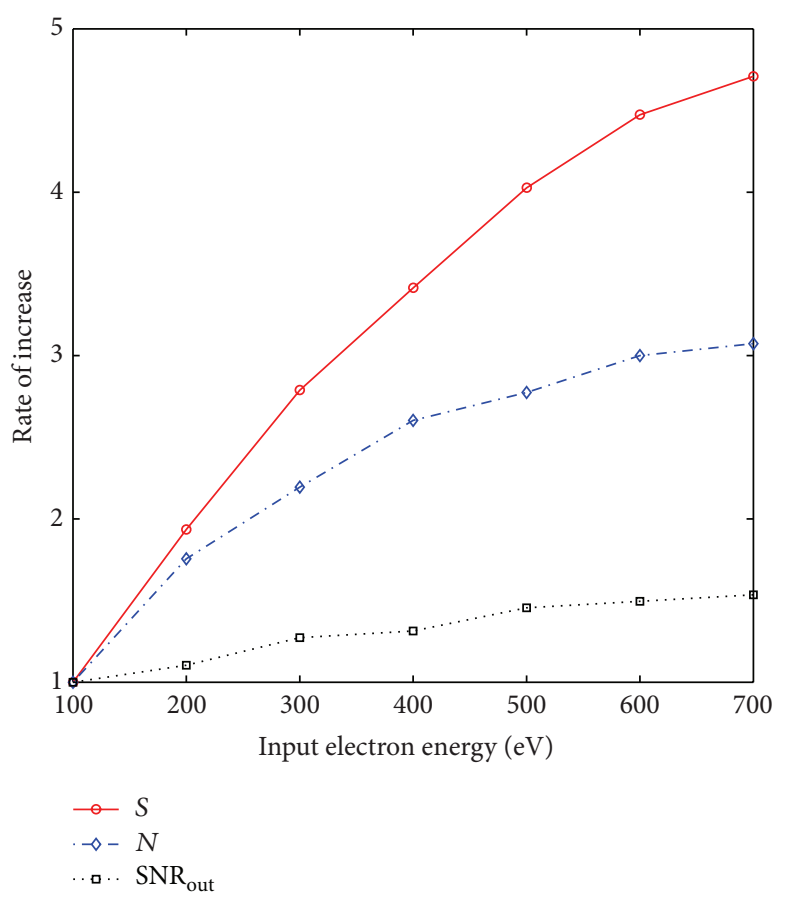

FIgURE 5: The rate of increase in $S, N$, and $\mathrm{SNR}_{\text {out }}$ of MCP at different input electron energies. 
TABLE 3: Noise factor of MCP at different input electron energies.

\begin{tabular}{lccc}
\hline Input electron energy $(\mathrm{eV})$ & $\mathrm{SNR}_{\text {in }}$ & $\mathrm{SNR}_{\text {out }}$ & $N_{F}$ \\
\hline 100 & 48.54 & 24.66 & 1.968 \\
200 & 48.54 & 27.22 & 1.783 \\
300 & 48.54 & 31.38 & 1.547 \\
400 & 48.54 & 32.38 & 1.499 \\
500 & 48.54 & 35.88 & 1.353 \\
600 & 48.54 & 36.86 & 1.317 \\
700 & 48.54 & 37.86 & 1.282 \\
\hline
\end{tabular}

TABLE 4: Noise factor of MCP at different voltages.

\begin{tabular}{lccc}
\hline Voltage across the MCP $(\mathrm{V})$ & $\mathrm{SNR}_{\text {in }}$ & SNR $_{\text {out }}$ & $N_{F}$ \\
\hline 600 & 48.54 & 28.53 & 1.701 \\
650 & 48.54 & 29.07 & 1.670 \\
700 & 48.54 & 29.38 & 1.652 \\
750 & 48.54 & 30.48 & 1.593 \\
800 & 48.54 & 32.46 & 1.495 \\
\hline
\end{tabular}

The striking feature of Table 3 is that $\mathrm{SNR}_{\text {out }}$ of $\mathrm{MCP}$ increases with increasing input electron energy, and thus its $N_{F}$ decreases as shown in Figure 4. It is well illustrated by the relationship between the rates of increase in signal and noise (Figure 5). Figure 5 shows that, as the input electron energy increases, $\mathrm{SNR}_{\text {out }}$ of MCP increase gradually since the rate of increase in signal is larger than that in noise. More specifically, with increasing input electron energy, the rate of increase in signal is substantially enhanced because more electrons have sufficient energies to generate secondary electrons, and the noise increases simultaneously to a certain degree. The increase in noise is mainly because the electrons scattered by impacting channel boundaries have obtained higher energies and been multiplied as the input electron energy increases. However, it should be noted that the rates of increase in signal and in noise are inclined to saturate when the input electron energy increases to a certain value. In addition, if primary event is too energetic, secondary electrons will be generated too deeply within the channel wall and fail to escape into the channel and be multiplied. Conversely, if the primary event has too little energy, secondary electrons will not be generated. Therefore, an appropriate input electron energy is important for the operation of MCP in image intensifier tubes. In general, for the unfilmed MCP and filmed MCP, the appropriate input electron energies are $400 \mathrm{eV}$ and $600 \mathrm{eV}$, respectively.

3.3.4. Noise Factor of MCP at Different Voltages. In order to study the effect of the voltage across the MCP on $N_{F}$, by varying the voltage in the range of $600-800 \mathrm{~V}$, we have measured $N_{F}$ of MCP on condition that the filament voltage of electron gun is $-400 \mathrm{~V}$ and the difference in voltage between the output end of MCP and phosphor screen retains 200 V. Table 4 and Figures 6 and 7 show the measurement results, the effect of voltage across the MCP on $N_{F}$, and the rate of increase in $S, N$, and $\mathrm{SNR}_{\text {out }}$, respectively.

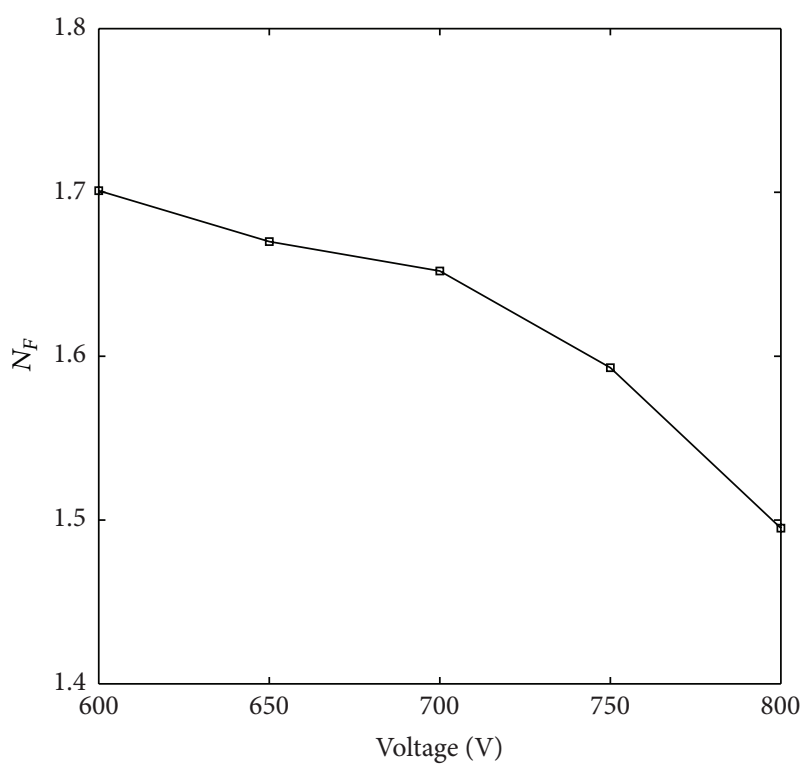

Figure 6: $N_{F}$ of MCP at different voltages.

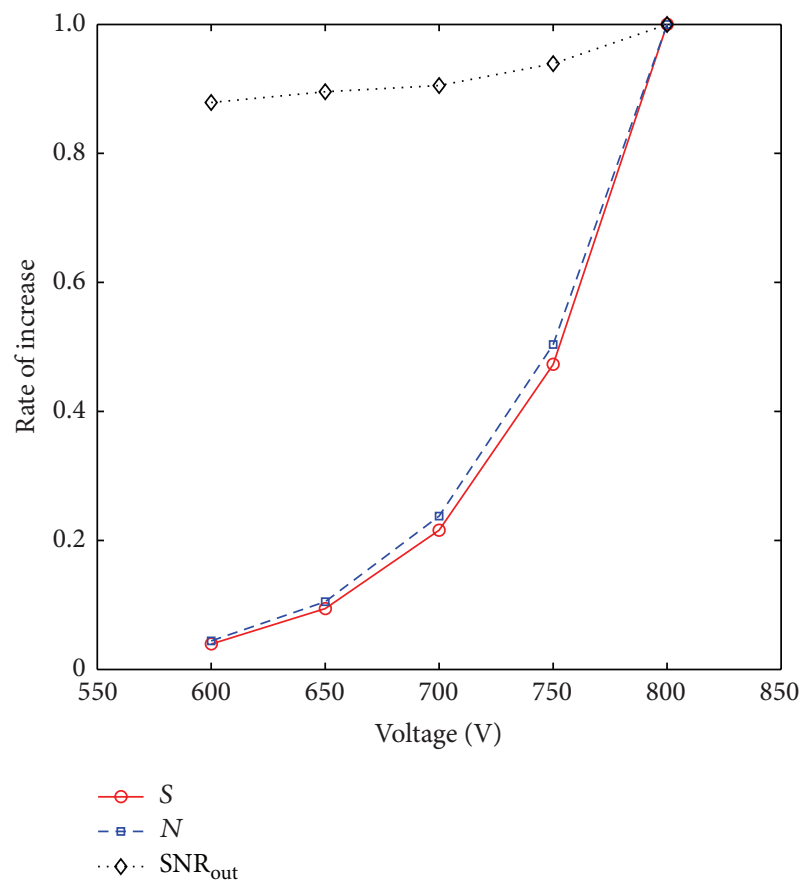

FIGURE 7: The rate of increase in $S, N$, and $\mathrm{SNR}_{\text {out }}$ of $\mathrm{MCP}$ at different voltages.

As shown in Table 4 and Figure 6, $N_{F}$ of MCP decreases with increasing voltage across the MCP. Figure 7 shows that, as the voltage increases, the electron energy in the channel increases more rapidly and secondary electrons are accelerated faster with the aid of electric field, thereby enhancing the rate of multiplication in signal. But, at the same time, the noise accompanying the amplified signal, including the electron scattering noise, the photon scattering noise, and the dark background noise, is multiplied as well in MCP. Additionally, 
with increasing voltage across the MCP, the ion feedback noise increases. Fortunately, the ion feedback generated by the residual gas in channel wall has a relative little effect on signal through the treatment of electronic clearing brush and of outgassing. Thus, the increase of voltage across the MCP raises $\mathrm{SNR}_{\text {out }}$ slightly and then reduces $N_{F}$ on a limited basis.

As discussed above, compared with results obtained by constant filament current, the values of $\mathrm{SNR}_{\text {in }}, \mathrm{SNR}_{\text {out }}$, and $N_{F}$ with varying filament current generated by the electron beam incorporating noise-excitation are more objective. Furthermore, it is shown that not only can the removal of IBF reduce $N_{F}$ of MCP, but also larger open area ratio, higher input electron energy, and higher voltage across the MCP can reduce $N_{F}$, thereby improving the noise characteristics of $\mathrm{MCP}$ and the image quality of image intensifier. However, in practice, the removal of IBF will reduce the lifetime of photocathode significantly. In other words, the IBF extends the lifetime of the image tube but at the expense of increased $N_{F}$. The higher open area ratio will be restricted to the state of the art in fabrication, although the input open area ratio of greater than $72 \%$ has been achieved. As the incident electron energy increases, the extent of ion feedback will become stronger, and then the Cs-O layer on photocathode surface will be damaged to markedly reduce the lifetime of photocathode. Moreover, the operational life characteristics of the MCP will be impacted with increasing voltage. Therefore, to achieve an optimum overall performance of image intensifier, it is necessary that a compromise between ion barrier film, open area ratio of MCP, input electron energy, and voltage across the MCP must be made.

\section{Conclusions}

With the method using the sine random signal with Poisson distribution as the noise-excitation for electron source, the noise characteristics of MCP have been effectively evaluated by determining its $N_{F}$. The measurement results show that $N_{F}$ of filmed MCP is lower than 1.8 and that the noise characteristics of unfilmed MCP are improved evidently, as the open area ratio and the input electron energy are $72 \%$ and $400 \mathrm{eV}$, respectively. In addition, larger open area ratio, higher input electron energy, and higher voltage across the MCP all can reduce effectively $N_{F}$, which are in good agreement with corresponding theory. For the IBF, its optimum thickness should be taken fully into account. Nevertheless, it should be emphasized that the above measures reducing $N_{F}$ of MCP are on a limited basis. Hence, a compromise among these parameters must be reached, for achieving the overall performance of an image intensifier.

\section{Conflict of Interests}

The authors declare that there is no conflict of interests regarding the publication of this paper.

\section{Acknowledgments}

The authors thank Huimin Zhao, Ruishan Bai, and Jian Liu for their helpful discussions. This work is supported by the
National Natural Science Foundation of China (Grant no. 61301023) and by the Postdoctoral Science Foundation of China (Grant no. 2014M560424).

\section{References}

[1] D. J. Ruggieri, "Microchannel plate imaging detectors," in Proceedings of the 13th Scintillation and Semiconductor Symposium, pp. 74-84, Washington, DC, USA, February 1972.

[2] R. L. Bell, "Noise figure of the MCP image intensifier tube," IEEE Transactions on Electron Devices, vol. 22, no. 10, pp. 821-829, 1975.

[3] J. C. Lupo, "The theory and measurement of the signal-tonoise ratio of second generation image intensifiers," Optica Acta: International Journal of Optics, vol. 19, no. 8, pp. 651-661, 1972.

[4] H. K. Pollehn, "Evaluation of image intensifiers," Optical Engineering, vol. 21, no. 1, pp. 34-37, 1982.

[5] I. P. Csorba, Image Tubes, Howard W. Sams \& Co., Indianapolis, Ind, USA, 1985.

[6] Y. Y. Wei and Z. M. Tao, "Theoretical analysis of MCP noise factor," Journal of Electronics, vol. 15, no. 6, pp. 655-658, 1993.

[7] L. Liu, Z. J. Huang, T. Pan, and Y. S. Qian, "Measurement device for noise factor of microchannel plate," Applied Optics, vol. 51, no. 7, pp. 883-887, 2012.

[8] C. Clamberlini, G. Longobardi, P. L. Ramazz et al., "New approach to noise factor measurement of imaging devices," Optical Engineering, vol. 33, no. 3, pp. 845-849, 1994.

[9] A. B. Berkin and V. V. Vasilev, "How the interaction of the channels of a microchannel plate affects the image contrast," Journal of Optical Technology, vol. 75, no. 5, pp. 338-340, 2008.

[10] Y. J. Cheng, F. Shi, H. Guo et al., "Effect of MCP parameters on resolution of image intensifier," Journal of Applied Optics, vol. 31, no. 2, pp. 292-296, 2010.

[11] N. Chen, W. S. Le, G. Q. Long et al., "Manufacture technology of microchannel plate with high resolution," Laser \& Optoelectronics Progress, vol. 47, no. 6, Article ID 062201, 5 pages, 2010.

[12] B. R. Sandel, A. L. Broadfoot, and D. E. Shemansky, "Microchannel plate life tests," Applied Optics, vol. 16, no. 5, pp. 1435-1437, 1977.

[13] B. N. Laprade, S. T. Reihart, and M. Wheeler, "Low-noise-figure microchannel plate optimized for Gen III image intensification systems," in Electron Image Tubes and Image Intensifiers, vol. 1243 of Proceedings of SPIE, pp. 162-172, Santa Clara, Calif, USA, February 1990. 

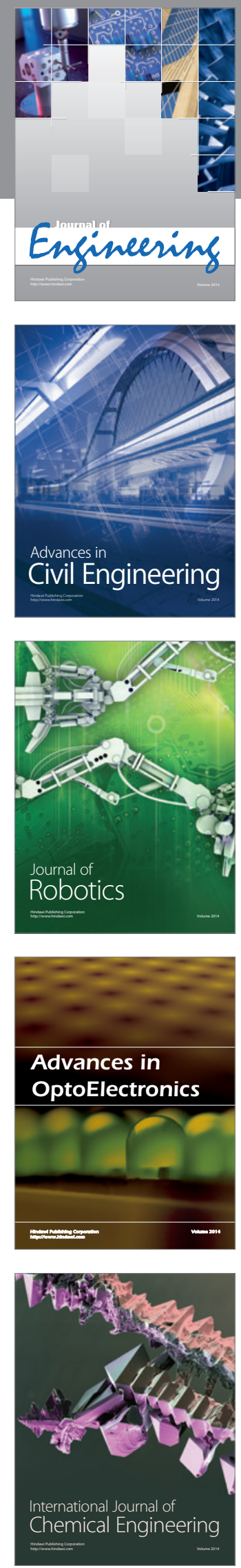

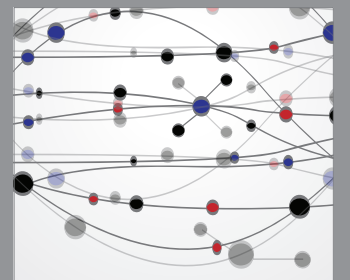

The Scientific World Journal
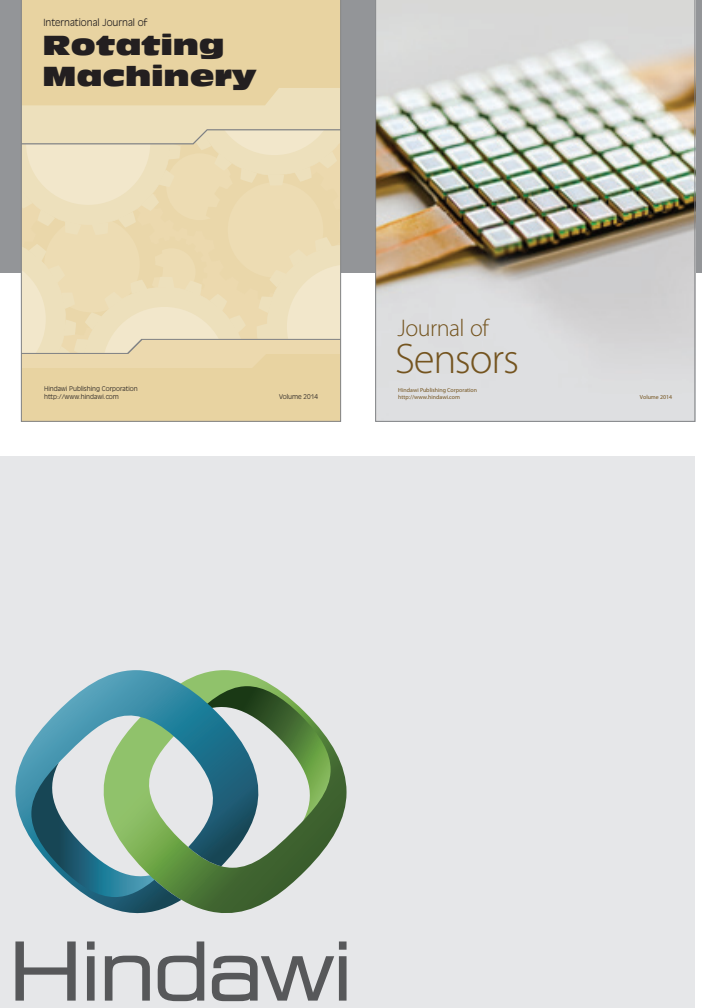

Submit your manuscripts at http://www.hindawi.com
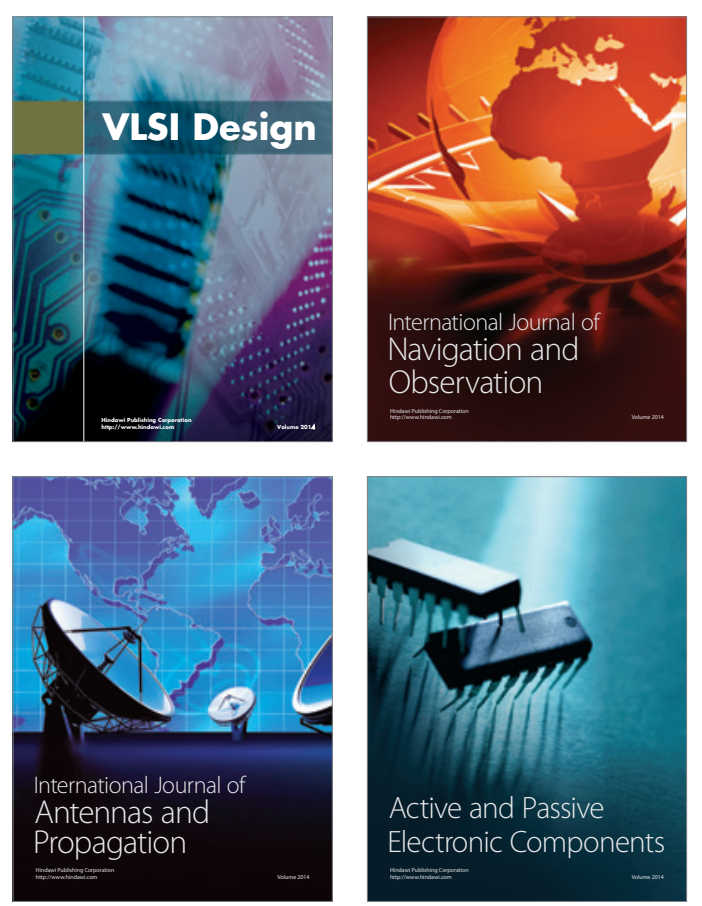
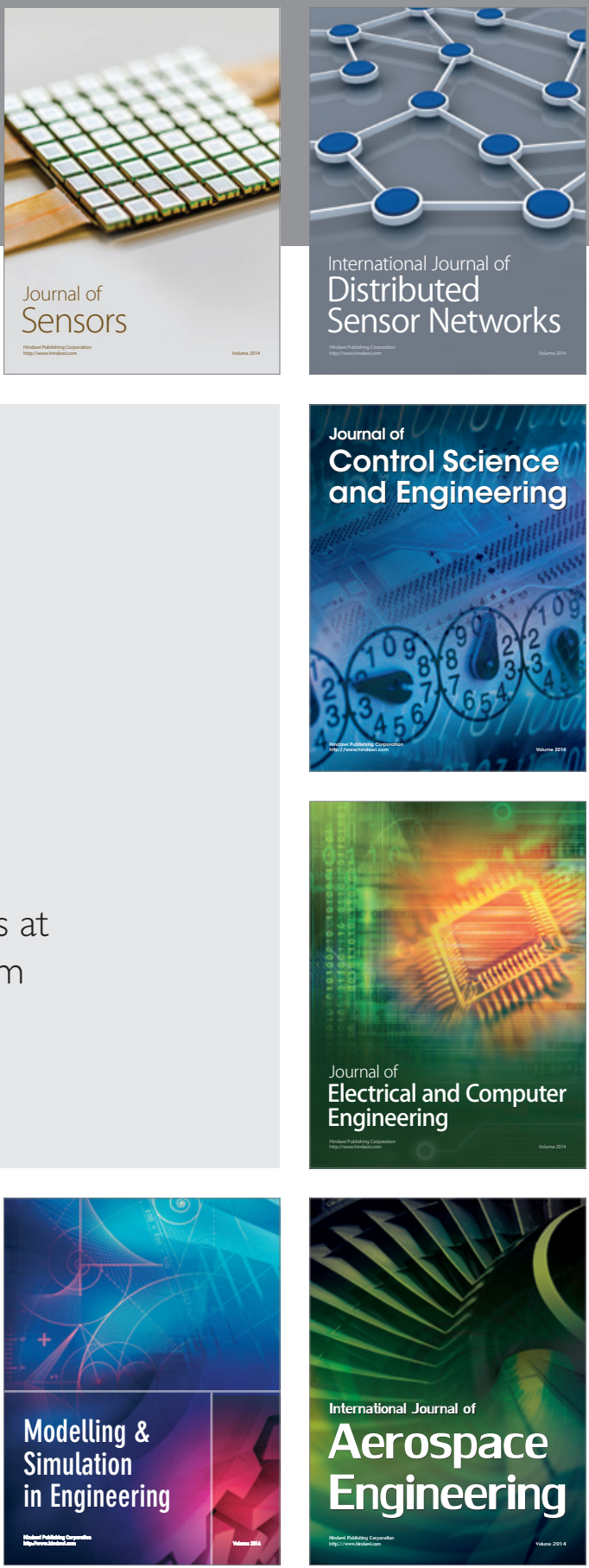

Journal of

Control Science

and Engineering
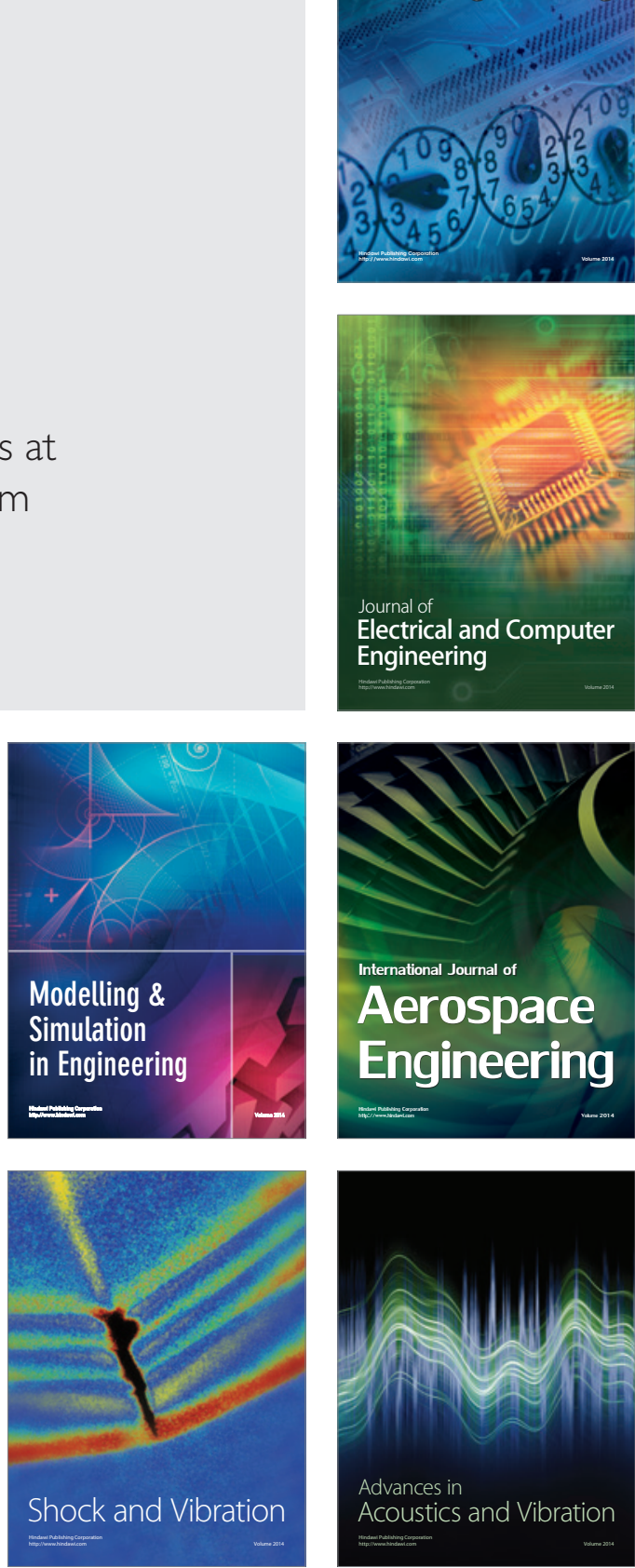